\title{
CORRECTIONS
}

\section{Six in 10 doctors in Scotland plan to vote against independence}

In this News story (BMJ 2014;349:g5072, doi:10.1136/bmj. g5072), we incorrectly stated that the name of the Labour Member of the Scottish Parliament (MSP) in the debate was Andrew Robertson. In fact, his name was Richard Simpson.
In addition, the article incorrectly described the two MSPs as "ministers" when they are, in fact, Members of the Scottish Parliament.

Cite this as: BMJ 2014;349:95235

๑) BMJ Publishing Group Ltd 2014 